p1130 Deep

thoughts: The Dalai

Lama's lecture on the

science of meditation

has provoked protests.

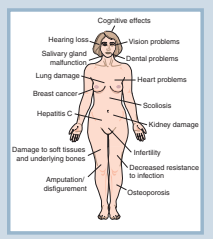

cures: Treatments for

childhood cancers

have long-term

consequences. p1132 Accursed p1134 Science and the city: Marie Filbin heads a lab in style at a small New York college.

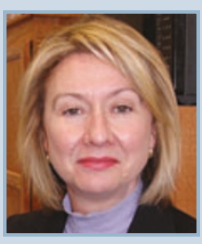

\section{Mental health research emerges from hurricane ruin}

When Michael Charness arrived at a makeshift Texas hospital in October, ready to treat those who had fled their homes after Hurricanes Katrina and Rita, he was quickly overwhelmed by the number of people with behavioral issues. "Soon after we began to work with them, we began to see the magnitude and volume of mental health problems," says Charness, chief of staff for the Veterans Administration Boston Healthcare System.

Solid numbers are unlikely to be available for months, or even years, but according to informal surveys of people in the facility, about half have mental health problems, and nearly one-third show signs of post-traumatic stress disorder (PTSD). "Even if only a small percentage of hurricane victims go on to develop new mental health problems, the number could be staggering because of the large scale of the disaster," says Farris Tuma, head of disaster research at the US National Institute of Mental Health (NIMH).

In the aftermath of disasters, many people experience intrusive thoughts and insomnia, but few suffer long-term consequences. Unfortunately, Katrina featured many of the ingredients that trigger persistent disorders. "Effects are more likely to linger when severe trauma is coupled with community destruction," says Fran Norris, a psychologist at Dartmouth Medical School.

Based on data from 132 different disasters over the past 24 years, Norris has found that risk factors for PTSD include injuries, life-threatening situations, bereavement, displacement and financial loss. Minorities are also at greater risk.

Researchers plan to study efforts to get 'psychological first aid' to thousands of victims, many of whom will recover on their own, as well as programs for people who develop psychiatric problems, such as depression and PTSD. The NIMH will support some of these efforts with RAPID research grants.

A primary challenge will be to find the small proportion of people most likely to suffer long-term problems. "We'd like to be able to identify people at risk with gene expression or biomarkers, like you do for people who are at and people from lower socioeconomic class risk for a stroke," says NIMH director Thomas Insel.

No such clinical tests are available as yet, but scientists have found some promising candidates. Researchers at the Hadassah University Hospital in Jerusalem measured gene expression of a subgroup of blood cells

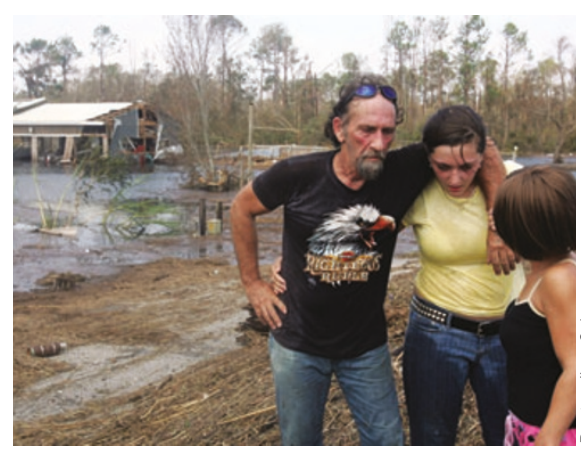

Dark water: Hurricane Katrina is likely to cause long-term mental health problems. in emergency room patients who came to the hospital after trauma, such as terrorist attacks. They found that gene expression signatures recorded during the emergency visit and four months after predicted which individuals met diagnostic criteria for PTSD (Mol. Psychiatry 10, 500; 2005). The results could aid early detection and intervention efforts, they say.

However, further research on these types of biomarkers is unlikely to take place with victims of Katrina, because of ethical concerns associated with asking people for blood samples so soon after a horrific event, says Insel.

In the meantime, Ron Kessler, a healthcare researcher at Harvard Medical School, plans to follow 1,000 people displaced by the hurricane for at least two years to track the ongoing stresses and psychological effects, as well as their access to medical care.

Surveys such as these will also help determine how treatment patterns have changed since $9 / 11$ and other disasters.

Emily Singer, Boston

\section{Katrina sets off maternal stress studies}

Hurricane Katrina created a unique opportunity to study a large population of pregnant women who experienced the same stressful event, says Pathik Wadhwa, a behavioral medicine researcher at the University of California at Irvine, who studies how maternal stress affects an unborn baby's health.

The results could help scientists understand the short- and long-term effects of maternal stress, as well as the stages of pregnancy when stress is most harmful. Previous research in humans has shown that extreme maternal stress is linked to premature birth and babies with low birth weight. Animal studies also suggest that stress has wide-ranging impacts on processes such as the development of the immune system and the brain.

Wadhwa and his colleagues are working with the US Federal Emergency Management Agency to compile a registry of pregnant women who survived the disaster in order to track their health over time. They plan to assess the level of stress that these women experienced during the disaster, such as if they lost their homes or were evacuated, and to evaluate their babies' health and complications during pregnancy and birth. Because women were in different stages of pregnancy when the hurricane struck, researchers can try to determine which periods of fetal development are most vulnerable to stress.

Much of the data can be collected later in time, which eases ethical concerns about asking women who are still recovering from the disaster to participate in a study, says Wadhwa. For example, researchers can use medical records compiled during birth to assess pregnancy complications and infant health.

Researchers are still working out details of the study, but they ultimately hope to follow some children for several years. They might also take DNA samples to assess how gene-environment interactions affect the impact of stress.

Emily Singer, Boston 